Pacific Journal of Mathematics

CONVEXITY THEOREMS FOR SUBCLASSES OF UNIVALENT 


\section{CONVEXITY THEOREMS FOR SUBCLASSES OF UNIVALENT FUNCTIONS}

\section{Herb Silverman}

We determine the radius of convexity of functions $f(z)$ for which $\operatorname{Re}\left\{f^{\prime}(z) / \phi^{\prime}(z)\right\}>\beta$, where $\phi(z)$ is convex of order $\alpha(0 \leqq \alpha \leqq 1)$. We also find bounds for $\left|\arg f^{\prime}(z)\right|$. All result are sharp.

1. Introduction. Let $S$ be the class of normalized univalent functions analytic in the unit disk. Let $K(\alpha)$ denote the subclass of $S$ consisting of functions $\phi(z)$ for which

$$
\operatorname{Re}\left\{1+\frac{z \phi^{\prime \prime}(z)}{\phi^{\prime}(z)}\right\} \geqq \alpha \quad(0 \leqq \alpha \leqq 1) .
$$

This class is called convex of order $\alpha$. We say that an analytic function $f(z)=z+a_{2} z^{2}+\cdots$ is in the class $C(\alpha, \beta)$ if there exists a function $\phi(z) \in K(\alpha)$ such that

$$
\operatorname{Re}\left\{\frac{f^{\prime}(z)}{\phi^{\prime}(z)}\right\}>\beta \quad(0 \leqq \beta<1,|z|<1) .
$$

This class was defined by Libera [5]. Kaplan [3] showed that $C(0,0)$, the class of close-to-convex functions, is univalent. Since $C(\alpha, \beta) \subset C(0,0)$, we see that $C(\alpha, \beta)$ is a subclass of $S$.

Denote by $P_{\beta}$ the functions $p(z)$ that are analytic in $|z|<1$ and satisfy there the conditions

$$
p(0)=1 \text { and } \operatorname{Re} p(z)>\beta
$$

and set $P_{0}=P$. It is well known that a function $q(z)$ is in $P_{\beta}$ if and only if there exists a function $p(z) \in P$ such that

$$
\begin{gathered}
q(z)=(1-\beta) p(z)+\beta=\frac{p(z)+h}{1+h}, \text { where } \\
h=\frac{\beta}{1-\beta} .
\end{gathered}
$$

Thus if $f(z) \in K(\alpha, \beta)$, then we may write

$$
f^{\prime}(z)=\phi^{\prime}(z) \frac{p(z)+h}{1+h}
$$


where $\phi(z) \in K(\alpha), p(z) \in P$, and $h$ is defined by (1). Taking logarithmic derivatives in (2), we find that

$$
1+\frac{z f^{\prime \prime}(z)}{f^{\prime}(z)}=1+\frac{z \phi^{\prime \prime}(z)}{\phi^{\prime}(z)}+\frac{z p^{\prime}(z)}{p(z)+h}
$$

It is our purpose in this paper to determine the radius of convexity for the class $C(\alpha, \beta)$. Note, for $|z|=r$, that (3) yields

$$
\begin{aligned}
\min _{f \in C(\alpha, \beta)} \operatorname{Re}\left\{1+\frac{z f^{\prime \prime}(z)}{f^{\prime}(z)}\right\}= & \min _{\phi \in K(\alpha)} \operatorname{Re}\left\{1+\frac{z \phi^{\prime \prime}(z)}{\phi^{\prime}(z)}\right\} \\
& +\min _{p \in P} \operatorname{Re}\left\{\frac{z p^{\prime}(z)}{p(z)+h}\right\} .
\end{aligned}
$$

In [5], Libera found a disk $|z|<r$ in which $f(z) \in C(\alpha, \beta)$ is convex. His method essentially consisted of utilizing the inequaltiy

$$
\min _{f \in C(\alpha, \beta)} \operatorname{Re}\left\{1+\frac{z f^{\prime \prime}(z)}{f^{\prime}(z)}\right\} \geqq \min _{\phi \in K(\alpha)} \operatorname{Re}\left\{1+\frac{z \phi^{\prime \prime}(z)}{\phi^{\prime}(z)}\right\}-\max _{p \in P}\left|\frac{z p^{\prime}(z)}{p(z)+h}\right| .
$$

His result, however, was not sharp because for $|z|=r$,

$$
\min _{p \in P} \operatorname{Re}\left\{\frac{z p^{\prime}(z)}{p(z)+h}\right\} \geqq-\max _{p \in P}\left|\frac{z p^{\prime}(z)}{p(z)+h}\right| \text {, }
$$

with equality only when $h=0$. The function that he claimed to be extremal need not be in $C(\alpha, \beta)$. See [9]. It is known [1] that

$$
\min _{\substack{|z|=r \\ \phi \in K(\alpha)}} \operatorname{Re}\left\{1+\frac{z \phi^{\prime \prime}(z)}{\phi^{\prime}(z)}\right\}=\frac{1-(1-2 \alpha) r}{1+r}
$$

with equality for functions of the form

$$
\phi(z)= \begin{cases}\frac{1}{(1-2 \alpha) \epsilon}\left[\frac{1}{(1-\epsilon z)^{1-2 \alpha}}-1\right] & \left(\alpha \neq \frac{1}{2},|\epsilon|=1\right) \\ -\bar{\epsilon} \log (1-\epsilon z) & \left(\alpha=\frac{1}{2},|\epsilon|=1\right) .\end{cases}
$$

Thus, taking into account (4), the radius of convexity of $C(\alpha, \beta)$ is seen to be the smallest positive $r$ for which

$$
\frac{1-(1-2 \alpha) r}{1+r}+\min _{\substack{|z|=r \\ p \in P}} \operatorname{Re}\left\{\frac{z p^{\prime}(z)}{p(z)+h}\right\}=0 .
$$


In $\$ 2$ we will use a theorem of V. A. Zmorovič to find $\min _{|z|=r, p \in P} \operatorname{Re}\left\{z p^{\prime}(z) /(p(z)+h)\right\}$. In $\S 3$ we will determine the radius of convexity of $C(\alpha, \beta)$ and examine some of its consequences. Finally, in $\S 4$ we will find a sharp bound on $\left|\arg f^{\prime}(z)\right|$ for $f(z) \in C(\alpha, \beta)$.

2. Consequences of Zmorovič's theorem. The following theorem is due to V. A. Zmorovič [11].

THEOREM A. Let $\Psi(w, W)=M(w)+N(w) W$, where $M(w)$ and $N(w)$ are defined and are finite in the half plane $\operatorname{Re}\{w\}>0$. Set

$$
\begin{gathered}
w=\lambda_{1} \frac{1+z_{1}^{m}}{1-z_{1}^{m}}+\lambda_{2} \frac{1+z_{2}^{m}}{1-z_{2}^{m}}, \\
W=\lambda_{1} \frac{2 m z_{1}^{m}}{\left(1-z_{1}^{m}\right)^{2}}+\lambda_{2} \frac{2 m z_{2}^{m}}{\left(1-z_{2}^{m}\right)^{2}},
\end{gathered}
$$

where $z_{1}$ and $z_{2}$ are any points on the circle $|z|=r<1, m$ is a positive integer, $\lambda_{1} \geqq 0, \lambda_{2} \geqq 0$, and $\lambda_{1}+\lambda_{2}=1$. Then the function $\Psi(w, W)$ can be put in the form

$$
\Psi(w, W)=M(w)+\frac{m}{2}\left(w^{2}-1\right) N(w)+\frac{m}{2}\left(\rho^{2}-\rho_{0}^{2}\right) N(w) e^{2 \imath \psi},
$$

where

$$
\begin{aligned}
\frac{1+z_{k}^{m}}{1-z_{k}^{m}} & =a+\rho e^{i \psi_{k}} & & (k=1,2), \\
w & =a+\rho_{0} e^{i \psi_{0}} & & \left(0 \leqq \rho_{\theta} \leqq \rho\right), \\
a & =\frac{1+r^{2 m}}{1-r^{2 m}}, & \rho & =\frac{2 r^{m}}{1-r^{2 m}}, \quad e^{i \psi}=i e^{i\left(\psi_{1}+\psi_{2}\right) / 2} .
\end{aligned}
$$

Also,

$$
\begin{aligned}
\min & \operatorname{Re}\{\Psi(w, W)\} \equiv \Psi_{\rho}(w) \\
& =\operatorname{Re}\left\{M(w)+\frac{m}{2}\left(w^{2}-1\right) N(w)\right\}-\frac{m}{2}|N(w)|\left(\rho^{2}-\rho_{0}^{2}\right) .
\end{aligned}
$$

This minimum is reached when

$$
\exp [i(2 \psi+\arg N(w)]=-1
$$

The importance of this formidable theorem lies in the fact that the minimum of $\operatorname{Re} \Psi(w, W)$ in the disk $|w-a| \leqq \rho$ depends only on the two variables $\operatorname{Re} w$ and $\operatorname{Im} w$, as can be seen by (6), and not on $W, \lambda_{1}$, or $\lambda_{2}$. 
I would like to thank the referee for pointing out that the following theorem may be found in [12]. For completeness we include a more detailed proof of this useful result.

Theorem 1. Suppose $p(z) \in P, h$ is defined by (1), and $a$ is defined as in Theorem A. Then

$$
\operatorname{Re}\left\{\frac{z p^{\prime}(z)}{p(z)+h}\right\} \geqq\left\{\begin{array}{cc}
-\frac{2 r}{(1+r)[(1+h-(1-h) r]} & \left(0 \leqq r \leqq r_{\beta}\right) \\
2 \sqrt{h^{2}+a h}-a-2 h & \left(r_{\beta}<r<1\right),
\end{array}\right.
$$

where $r_{\beta}$ is the unique root of the equation $(1-2 \beta) r^{3}-3(1-2 \beta) r^{2}+3 r-$ $1=0$ in the interval $(0,1]$. This result is sharp.

Proof. Set $M(w)=0, N(w)=1 /(w+h), m=1$, and $w=p(z)=p$ in Theorem $A$, and note that $W=z p^{\prime}(z)$. Thus $\Psi(w, W)=\Psi\left(p, z p^{\prime}\right)=$ $z p^{\prime}(z) /(p(z)+h)$ and, in view of $(6)$,

$$
\operatorname{Re}\left\{\frac{z p^{\prime}(z)}{p(z)+h}\right\} \geqq \Psi_{\rho}(p)=\frac{1}{2} \operatorname{Re}\left[\frac{p^{2}-1}{p+h}-\frac{\rho^{2}-\rho_{0}^{2}}{|p+h|}\right] .
$$

Since $|p-a|=\rho_{0} \leqq \rho$, we may set $p=a+\xi+i \eta, \rho_{0}^{2}=\xi^{2}+\eta^{2}$, and $R=|p+h|$. Then

(8) $\operatorname{Re} \frac{p^{2}-1}{p+h}=\frac{|p|^{2}(a+\xi)-(a+\xi+h)+h\left[(a+\xi)^{2}-\eta^{2}\right]}{R^{2}}$

$$
\begin{aligned}
& =\frac{(a+\xi+h)\left[R^{2}-\left(h^{2}+2 h(a+\xi)+1\right)\right]-2 h \eta^{2}}{R^{2}} \\
& =\frac{(a+\xi+h)\left[R^{2}-2 h(a+\xi+h)+\left(h^{2}-1\right)\right]-2 h \eta^{2}}{R^{2}} \\
& =(a+\xi+h)-2 h+\frac{\left(h^{2}-1\right)(a+\xi+h)}{R^{2}} .
\end{aligned}
$$

A substitution of (8) into (7) gives

$$
\Psi_{\rho}(p)=\frac{a+\xi+h}{2}-h+\frac{\left(h^{2}-1\right)(a+\xi+h)}{2 R^{2}}-\frac{\rho^{2}-\xi^{2}-\eta^{2}}{2 R} .
$$

We now wish to minimize $\Psi_{\rho}(p)$ as a function of $\eta$. A differentiation shows that

$$
\frac{\partial \psi_{\rho}}{\partial \eta}=\frac{\eta}{2} \frac{S(\xi, \eta)}{R^{4}}
$$


where

$$
\begin{aligned}
S(\xi, \eta) & =\left[\xi^{2}+4(a+h) \xi+\rho^{2}+\eta^{2}+2(a+h)^{2}\right] R-2\left(h^{2}-1\right)(\xi+a+h) \\
11) & \geqq\left[\xi^{2}+4(a+h) \xi+\rho^{2}+2(a+h)^{2}-2\left(h^{2}-1\right)\right](\xi+a+h) .
\end{aligned}
$$

But the last expression in (11) is an increasing function of $\xi$ in the interval $[-\rho, \rho]$. Hence

$$
S(\xi, \eta) \geqq S(-\rho, \eta)=2\left[(a-\rho)^{2}+2 h(a-\rho)+1\right](a+h-\rho)>0 .
$$

We thus see from $(10)$ that $\Psi_{\rho}(\xi, \eta)$ is minimized on every chord $\xi=$ constant of the circle $\xi^{2}+\eta^{2}=\rho_{0}^{2}$ at the point $\eta=0$. Therefore the minimum of $\Psi_{\rho}(\xi, \eta)$ in the disk $\xi^{2}+\eta^{2} \leqq \rho^{2}$ occurs somewhere on the diameter $\eta=0$. Setting $\eta=0$ in (9) and noting that $R=a+\xi+h$, we have

$$
\Psi_{\rho}(p) \geqq \Psi_{\rho}(\xi, 0)=l(R)=\frac{R}{2}-h+\frac{h^{2}+\xi^{2}-\rho^{2}-1}{2 R} .
$$

Using the identities $\xi=R-(a+h)$ and $\rho^{2}=a^{2}-1$ in (12), we get

$$
l(R)=R+\frac{h^{2}+a h}{R}-(a+2 h) .
$$

We must now determine the minimum of $l(R)$ for $R$ in the interval $[a+h-\rho, a+h+\rho]$. A differentiation of (13) shows that $l(R)$ assumes its minimum at

$$
R_{0}=\sqrt{h^{2}+a h}
$$

as long as

$$
a+h-\rho \leqq R_{0} \leqq a+h+\rho .
$$

The right hand inequality in (15) is always true, but the left hand inequality will not hold when $h$ (and consequently $\beta$ ) is small. In the latter case, $l(R)$ assumes its minimum at the point

$$
R_{1}=a+h-\rho .
$$

Substituting (14) and (16), respectively, into (13), we find

$$
l\left(R_{0}\right)=2 \sqrt{h^{2}+a h}-(a+2 h)
$$




$$
l\left(R_{1}\right)=\frac{\rho^{2}-a \rho}{a+h-\rho}=-\frac{2 r}{(1+r)[1+h-(1-h) r]} .
$$

As $\beta$ increases, the transition from $l\left(R_{1}\right)$ to $l\left(R_{0}\right)$ occurs at the point where $R_{0}=R_{1}$. But $R_{0}=R_{1}$ when $h^{2}+a h=(a-\rho+h)^{2}$, or in terms of $r$, when the polynomial equation

$$
t(r)=(1-2 \beta) r^{3}-3(1-2 \beta) r^{2}+3 r-1
$$

has a root in the interval $(0,1]$. Note that

$$
t^{\prime}(r)=3\left[(1-2 \beta) r^{2}-2(1-2 \beta) r+1\right]>0 \quad(0<r<1)
$$

so that $t(r)$ is increasing. Further, $t(0)=-1$ and $t(1)=4 \beta$ so that $t(r)$ has a unique root in the interval $(0,1]$. This completes the proof.

Equality holds in (18) for $p(z)=(1+z) /(1-z)$, and in (17) for

$$
p(z)=\frac{1}{2}\left[\frac{1+z e^{-i \theta_{0}}}{1-z e^{-i \theta_{0}}}+\frac{1+z e^{i \theta_{0}}}{1-z e^{i \theta_{0}}}\right]=\frac{1-z^{2}}{1-2 z \cos \theta_{0}+z^{2}},
$$

where $\cos \theta_{0}$ is defined by the equation

$$
h+\left(1-r_{0}^{2}\right)\left(1-2 r_{0} \cos \theta_{0}+r_{0}^{2}\right)^{-1}=R_{0} \quad\left(r_{0}=l\left(R_{0}\right)\right) .
$$

3. Radius of convexity theorems. We may now use Theorem 1 to prove

THEOREM 2. Suppose $r_{\beta}$ is the unique root of

$$
t(r)=(1-2 \beta) r^{3}-3(1-2 \beta) r^{2}+3 r-1
$$

in the interval $(0,1] . \quad$ Set

$$
r(\alpha, \beta)=\frac{1}{2-\alpha-2 \beta+\sqrt{\alpha^{2}-2 \alpha+4 \beta^{2}-6 \beta+3}} .
$$

Then the radius of convexity of $C(\alpha, \beta)$ is $r(\alpha, \beta)$ when $0<r(\alpha, \beta) \leqq r_{\beta}$, and is otherwise the smallest root greater than $r_{\beta}$ of the polynomial equation

$$
\begin{aligned}
v(r)= & {\left[\alpha^{2}-\beta\left(\alpha^{2}+2 \alpha-1\right)\right] r^{4}-2(1-\alpha)(\beta+\alpha \beta-\alpha) r^{3} } \\
& +\left[(1-\alpha)^{2}(1-\beta)+2 \alpha \beta\right] r^{2}+2 \beta(1-\alpha) r-\beta .
\end{aligned}
$$

This result is sharp for all $\alpha$ and $\beta$. 
Proof. An application of Theorem 1 to (5) shows that the radius of convexity of $C(\alpha, \beta)$ is the smallest positive root of

$$
\begin{cases}\frac{1-(1-2 \alpha) r}{1+r}-\frac{2 r}{(1+r)[(1+h)-(1-h) r]}=0 & \left(0 \leqq r \leqq r_{\beta}\right) \\ \frac{1-(1-2 \alpha) r}{1+r}+2 \sqrt{h^{2}+a h}-a-2 h=0 & \left(r_{\beta}<r<1\right),\end{cases}
$$

where $a$ is defined in Theorem A and $h$ is defined by (1). The first expression in (20) may be written as

$$
\frac{(1-2 \alpha)(1-2 \beta) r^{2}-2(2-\alpha-2 \beta) r+1}{(1+r)[(1+h)-(1-h) r]}=0,
$$

whose roots are

$$
\begin{aligned}
& \frac{(2-\alpha-2 \beta) \mp \sqrt{(2-\alpha-2 \beta)^{2}-(1-2 \alpha)(1-2 \beta)}}{(1-2 \alpha)(1-2 \beta)} \\
& \quad=\frac{1}{(2-\alpha-2 \beta) \pm \sqrt{\alpha^{2}-2 \alpha+4 \beta^{2}-6 \beta+3}}
\end{aligned}
$$

If both roots are positive, the minimum root is $r(\alpha, \beta)$. Similarly, a computation shows that $r^{*}$ is a root of the second expression in (20) if and only if it is a root of $v(r)$. This completes the proof.

The extremal function is of the form

$$
f(z)=\int_{0}^{z} \frac{1+(1-2 \beta) t}{(1-t)^{3-2 \alpha}} d t
$$

when $0<r(\alpha, \beta) \leqq r_{\beta}$, and is otherwise of the form

$$
f(z)=\int_{0}^{z} \frac{1-2 \beta \cos \theta_{0}+(2 \beta-1) t^{2}}{\left(1-2 t \cos \theta_{0}+t^{2}\right)(1-t)^{2(1-\alpha)}} d t,
$$

where $\cos \theta_{0}$ is defined by (19).

COROLLARY. If $0 \leqq \beta \leqq \frac{1}{10}$, then the radius of convexity of $C(\alpha, \beta)$ is $r(\alpha, \beta)$ for all $\alpha$.

Proof. We must show that $0<r(\alpha, \beta) \leqq r_{\beta}$ for $0 \leqq \alpha \leqq 1$ and $0 \leqq$ $\beta \leqq 1 / 10$. Note that $\partial t(r) / \partial \beta=2 r^{2}(3-r)$, so that $t(r)$ is an increasing function of $\beta$. This means that $r_{\beta}$ is a decreasing function of $\beta$. Set $A=\sqrt{\alpha^{2}-2 \alpha+4 \beta^{2}-6 \beta+3}$. Then 


$$
\frac{\partial}{\partial \alpha} r(\alpha, \beta)=\frac{A+1-\alpha}{S^{3}} \geqq 0 \quad(0 \leqq \alpha \leqq 1)
$$

and

$$
\frac{\partial}{\partial \beta} r(\alpha, \beta)=\frac{A+3-4 \beta}{A^{3}} \geqq 0 \quad\left(0 \leqq \beta \leqq \frac{3}{4}\right) .
$$

Thus $r(\alpha, \beta) \leqq r\left(1, \frac{1}{10}\right)$ for $0 \leqq \alpha \leqq 1$ and $0 \leqq \beta \leqq \frac{1}{10}$. The result follows upon observing that

$$
r\left(1, \frac{1}{10}\right)=\frac{1}{2} \quad \text { and } \quad t\left(\frac{1}{2}\right)=\frac{8}{10}\left(\frac{1}{8}\right)-\frac{24}{10}\left(\frac{1}{4}\right)+\frac{3}{2}-1=0 .
$$

REMARK. When $\beta=0$, we see that

$$
r(\alpha, 0)=\frac{1}{2-\alpha+\sqrt{\alpha^{2}-2 \alpha+3}} .
$$

In this case, Libera's result [5] is sharp.

We turn now to a distinguished subclass of $C(\alpha, \beta)$, and state the result as a separate theorem.

THEOREM 3. If $f(z) \in S$ with $\operatorname{Ref}^{\prime}(z)>\beta$, then $f(z)$ is convex in a disk of radius

$$
\begin{cases}\frac{1}{1-2 \beta+\sqrt{4 \beta^{2}-6 \beta+2}} & \left(0 \leqq \beta \leqq \frac{1}{10}\right) \\ \left(1+\sqrt{\frac{1-\beta}{\beta}}\right)^{-\frac{1}{2}} & \left(\frac{1}{10}<\beta<1\right) .\end{cases}
$$

This result is sharp.

Proof. Since $\phi(z)=z$ is the only function in $K(1)$, the class under consideration is $C(1, \beta)$ so that Theorem 2 may be applied. As we saw in the corollary to Theorem 2 ,

$$
r(1, \beta)=\frac{1}{1-2 \beta+\sqrt{4 \beta^{2}-6 \beta+2}} \leqq r_{\beta} \quad\left(0 \leqq \beta \leqq \frac{1}{10}\right)
$$

which gives the first part of the theorem.

Since $t\left(r_{\beta}\right)=0$ when $\alpha=1$ and $\beta=\frac{1}{10}$, the radius of convexity of $C(1, \beta)$ for $\beta>\frac{1}{10}$ is the only positive root of 


$$
\begin{aligned}
& (1-2 \beta) r^{4}+2 \beta r^{2}-\beta=0, \text { or } \\
& r^{2}=\frac{-\beta+\sqrt{\beta-\beta^{2}}}{1-2 \beta}=\frac{1}{1+\sqrt{\frac{1-\beta}{\beta}}} .
\end{aligned}
$$

This completes the proof.

REMARK. The cases $\beta=0$ and $\beta=\frac{1}{2}$ were proved, respectively, by MacGregor [6] and Hallenbeck [2].

\section{An argument theorem.}

THEOREM 4. If $f(z) \in C(\alpha, \beta)$, then

$$
\left|\arg f^{\prime}(z)\right| \leqq 2(1-\alpha) \sin ^{-1} r+\sin ^{-1}\left[\frac{2(1-\beta) r}{1+(1-2 \beta) r^{2}}\right] .
$$

This result is sharp.

Proof. We may write

$$
f^{\prime}(z)=\phi^{\prime}(z) q(z), \quad \text { where } \quad \phi(z) \in K(\alpha) \text { and } q(z) \in P_{\beta} .
$$

Hence

$$
\left|\arg f^{\prime}(z)\right| \leqq\left|\arg \phi^{\prime}(z)\right|+|\arg q(z)|
$$

But by a result of Pinchuk [8],

$$
\left|\arg \phi^{\prime}(z)\right| \leqq 2(1-\alpha) \sin ^{-1} r \quad(|z| \leqq r) .
$$

Since $\operatorname{Re} q(z)>\beta$, the function

$$
\omega(z)=\frac{(q(z)-\beta)-(1-\beta)}{(q(z)-\beta)+(1-\beta)}=\frac{q(z)-1}{q(z)-(2 \beta-1)}
$$

is analytic with $\omega(0)=0$ and $|\omega(z)|<1$ in $|z|<1$.

Thus by Schwarz's lemma,

$$
\left|\frac{q(z)-1}{q(z)-(2 \beta-1)}\right|<|z| \text { for }|z|<1
$$

Hence the values of $q(z)$ are contained in the circle of Apollonius whose diameter is the line segment from $(1+(2 \beta-1) r) /(1+r)$ to 
$(1-(2 \beta-1) r) /(1-r)$. The circle is centered at the point $\left(1+(1-2 \beta) r^{2}\right) /$ $\left(1-r^{2}\right)$ and has radius $(2(1-\beta) r) /\left(1-r^{2}\right)$. Thus $|\arg q(z)|$ attains its maximum at points where a ray from the origin is tangent to the circle, that is, when

$$
\arg q(z)= \pm \sin ^{-1} \frac{2(1-\beta) r}{1+(1-2 \beta) r^{2}}
$$

Substituting (22) and (23) into (21), the result follows.

Equality holds for functions of the form

$$
f(z)=\int_{0}^{z} \frac{1+(1-2 \beta) \eta t}{(1-\epsilon t)^{2(1-\alpha)}(1-\eta t)} d t
$$

with suitably chosen $\epsilon, \eta$, where $|\epsilon|=|\eta|=1$.

Remark. For $\alpha=\beta=0$, this reduces to

$$
\left|\arg f^{\prime}(z)\right| \leqq 2 \sin ^{-1} r+\sin ^{-1} \frac{2 r}{1+r^{2}}=2\left(\sin ^{-1} r+\tan ^{-1} r\right),
$$

a result of Krzyz [4].

TheORem 5. Suppose $f(z), g(z) \in C(\alpha, \beta)$. Then

$$
\lambda f(z)+(1-\lambda) g(z) \quad(0 \leqq \lambda \leqq 1)
$$

is univalent in a disk $|z|<r$, where $r$ is the smallest positive root of the equation

$$
2(1-\alpha) \sin ^{-1} r+\sin ^{-1}\left(\frac{2(1-\beta) r}{1+(1-2 \beta) r^{2}}\right)=\frac{\pi}{2} .
$$

This result is sharp.

Proof. In [7], MacGregor showed that the exact radius of univalence of convex linear combinations of a rotation and conjugation invariant subclass of $S$ is given by the supremum of the values of $r$ for which $\operatorname{Re} f^{\prime}(z)>0,|z|<r$, where $f(z)$ varies over all functions in the class. Since $K(\alpha)$ is rotation and conjugation invariant, see [10], so is $C(\alpha, \beta)$. That is, $f(z) \in C(\alpha, \beta)$ if and only if $\overline{f(\bar{z})}$ is in $C(\alpha, \beta)$. Since $\operatorname{Re} f^{\prime}(z)>0$ if and only if $\left|\arg f^{\prime}(z)\right|<\pi / 2$, the result follows from Theorem 4. 


\section{REFERENCES}

1. L. Brickman, D. J. Hallenbeck, T. H. MacGregor, and D. R. Wilkin, Convex hulls and extreme points of starlike and convex mappings, Trans. Amer. Math. Soc., 185 (1973), 413-428.

2. D. J. Hallenbeck, Convex hulls and extreme points of some families of univalent functions, Trans. Amer. Math. Soc., 192 (1974), 285-292.

3. W. Kaplan, Close-to-convex schlicht functions, Michigan Math. J., 1 (1952), 169-185.

4. J. Krzyz, On the derivative of close-to-convex functions, Colloq. Math., 10 (1963), 143-146.

5. R. J. Libera, Some radius of convexity problems, Duke Math. J., 31 (1964), 143-158.

6. T. H. MacGregor, Functions whose derivative has a positive real part, Trans. Amer. Math. Soc., 104 (1962), 532-537.

7. - The univalence of a linear combination of convex mappings, J. London Math. Soc., 44 (1969), 210-212.

8. B. Pinchuk, On starlike and convex functions of order $\alpha$, Duke Math. J., 4 (1968), 721-734.

9. H. Silverman, On a class of close-to-convex functions, Proc. Amer. Math. Soc., 36 (1972), $477-484$.

10. Linear combinations of convex mappings, Rocky Mountain J. Math., 5 (1975), 629-632.

11. V. A. Zmorovič, On bounds of convexity for starlike functions of order $\alpha$ in the circle $|z|<1$ and in the circular region $0<|z|<1$, Mat. Sb., 68 (110) (1965), 518-526; English transl., Amer. Math. Soc. Transl. (2) 80 (1969), 203-213.

12. - On the bounds of starlikeness and of univalence in certain classes of functions regular in the circle $|z|<1$, Amer. Math. Soc. Transl., (2) 80 (1967), 227-242.

Received September 12, 1975.

UNIVERSITY OF DELAWARE

Current address: College of Charleston Charleston, SC 29401 




\section{Pacific Journal of Mathematics}

Vol. 64, No. 1

May, 1976

Walter Allegretto, Nonoscillation theory of elliptic equations of order $2 n \ldots \ldots \quad 1$

Bruce Allem Anderson, Sequencings and starters.................. 17

Friedrich-Wilhelm Bauer, A shape theory with singular homology .......... 25

John Kelly Beem, Characterizing Finsler spaces which are

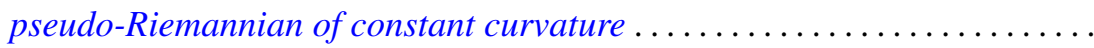

Dennis K. Burke and Ernest A. Michael, On certain point-countable

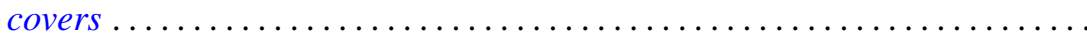

Robert Chen, A generalization of a theorem of Chacon ............... 93

Francis H. Clarke, On the inverse function theorem ................ 97

James Bryan Collier, The dual of a space with the Radon-Nikodým

property ....................................... 103

John E. Cruthirds, Infinite Galois theory for commutative rings ............ 107

Artatrana Dash, Joint essential spectra......................... 119

Robert M. DeVos, Subsequences and rearrangements of sequences in FK

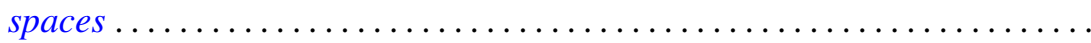

Geoffrey Fox and Pedro Morales, Non-Hausdorff multifunction generalization

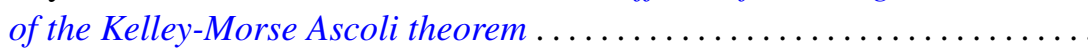

Richard Joseph Fleming, Jerome A. Goldstein and James E. Jamison, One

parameter groups of isometries on certain Banach spaces.............

Robert David Gulliver, II, Finiteness of the ramified set for branched

immersions of surfaces

Kenneth Hardy and István Juhász, Normality and the weak cb property ...... 167

C. A. Hayes, Derivation of the integrals of $L^{(q)}$-functions.

Frederic Timothy Howard, Roots of the Euler polynomials .

Robert Edward Jamison, II, Richard O'Brien and Peter Drummond Taylor, On

embedding a compact convex set into a locally convex topological vector space ....................................

Andrew Lelek, An example of a simple triod with surjective span smaller than span ...

Janet E. Mills, Certain congruences on orthodox semigroups

Donald J. Newman and A. R. Reddy, Rational approximation of $e^{-x}$ on the positive real axis.

John Robert Quine, Jr., Homotopies and intersection sequences ...

Nambury Sitarama Raju, Periodic Jacobi-Perron algorithms and fundamental units ....

Herbert Silverman, Convexity theorems for subclasses of univalent functions. . .

Charles Frederick Wells, Centralizers of transitive semigroup actions and endomorphisms of trees.........................

Volker Wrobel, Spectral approximation theorems in locally convex spaces ..................... 